\title{
Robótica educativa em Portugal - estado da arte
}

\section{Educational robotics in Portugal - state of the art}

\author{
João Marques, Vânia Ramos \\ AE de Figueiró dos Vinhos, AE de Sampaio
}

\begin{abstract}
Resumo
A programação e a robótica são duas áreas emergentes nas escolas portuguesas onde existem variados projetos que as mobilizam. Não se procura, na maioria destes projetos, formar futuros programadores, mas antes utilizar o ensino da programação e da robótica como metodologia para desenvolvimento de algumas competências: resolução de problemas, trabalho colaborativo, pensamento computacional, pensamento criativo, entre outras. Nos repositórios académicos existem diversos estudos que apresentam olhares sobre programas e projetos que integram a programação e a robótica em contexto educativo. Importa, pois, realizar uma leitura sistematizada sobre os estudos realizados e publicados e procurar identificar as vantagens e desvantagens da presença da programação e da robótica em contexto educativo.

Palavras-chave: robótica educativa, programação, pensamento computacional
\end{abstract}

\section{Abstract}

English Coding and Robotics are two emerging areas in portuguese schools where various projects are being implemented. In most projects, it is not intended to train future programmers but instead using the teaching of coding and robotics as a methodology to develop other competences such as: problem solving, collaboration, computational thinking, creative thinking, among others. In the academic repository there are several studies that present perspectives about programs and projects that gather coding and robotics in educational context. It is important to have a systematic reading of already made and published studies and try to identify the advantages and disadvantages of having coding and robotics present in the educational context.

Keywords: educational robotics, coding, computational thinking

\section{Introdução}

Este é um primeiro passo num processo mais longo de investigação sobre os contextos de implementação de projetos de robótica educativa em Portugal. Pretendemos conhecer não só os projetos, as suas dinâmicas, as metodologias utilizadas, de que forma foram avaliadas as estratégias e as atividades propostas, mas também quais as práticas e os contextos e que resultados produziram.

A utilização de robots em contexto educativo, quer seja em disciplinas ligadas diretamente à informática e à eletrónica, quer em contextos disciplinares diversos ou em atividades interdisciplinares, tem marcado o período recente da educação em Portugal. Os robots e a programação deixaram de ser de utilização exclusiva nas disciplinas de carácter mais técnico e passaram a utilizar-se em contextos escolares que vão do jardim-de-infância, à conceptualização e construção de teatros e danças robóticas, à utilização em contextos de aprendizagem de matemática, de física ou de conceitos ligados à programação, à automação e domótica. Pretendemos, nesse contexto fazer uma análise do que se tem publicado sobre esta temática nos últimos anos em Portugal em revistas científicas, em congressos e na forma de dissertações académicas.

Desta forma percorremos os repositórios das principais universidades portuguesas onde recolhemos comunicações em congressos e revistas científicas, capítulos de livros e dissertações de mestrado e doutoramento. Em cada um dos textos procurámos identificar as questões de investigação, as disciplinas envolvidas, as metodologias de investigação utilizadas e os principais resultados apresentados.

\section{Robótica educativa em Portugal}

A utilização de robots nas escolas portuguesas tem vindo a fazer-se de forma cada vez mais visível. Esta utilização permitida não apenas pelo continuado desenvolvimento de novas soluções tecnológicas, mas também pelo facto de no mercado existir cada vez mais oferta de robots especificamente pensados para contexto educativo e outros que, pela facilidade do seu desenvolvimento, são facilmente aplicáveis na escola. A progressiva baixa de preços destes equipamentos é também um fator importante para o sucesso desta caminhada (Coutinho, Ribeiro \& Costa, 2008). Não são ainda de descurar os projetos de investigação e de introdução da robótica na escola pela sua variedade de abordagens e diferentes dimensões.

Quando se fala em robótica educativa (RE), ou de robótica na escola, podemos ter diferentes abordagens. Assim como refere Oliveira (2004), "a Robótica aparece na escola e nas salas de aula essencialmente em três vertentes distintas:

i. a Robótica como disciplina tecnológica por si própria que merece uma abordagem autónoma;

ii.a Robótica como forma de ensinar/aprender conceitos relacionados com a programação;

iii. a Robótica utilizada como «um recurso pedagógico», ou seja como um meio para estimular a 
aprendizagem dos diversos conteúdos e competências em vários níveis de ensino".

A maioria dos estudos analisados abordam as segunda e terceira vertentes mencionadas. A primeira está presente, essencialmente, nos cursos profissionais e em disciplinas de carácter técnico. A segunda vertente está presente em disciplinas de programação onde a robótica pode ser mobilizada para tornar tangível os conceitos abordados e podendo os alunos, desta forma, validar ou tornar visível nos robots os conceitos e as aprendizagens realizadas. A terceira vertente é, talvez, a que tem vindo a dar mais visibilidade à robótica educativa. Como refere Ribeiro, Coutinho, \& Costa (2011b, p. 1500) “a RE apresenta diversas vantagens ao favorecer a interdisciplinaridade pelo facto de promover a integração de conceitos de diversas áreas, tais como: matemática, física, electricidade, electrónica, mecânica, arquitectura, ciências, história, geografia, artes, literacia, entre outras.

Esta ferramenta permite que sejam trabalhados conceitos de diversas disciplinas de uma forma prática, ao mesmo tempo que desenvolve competências e aspectos ligados ao planeamento e organização do trabalho".

A robótica pode constituir-se como um instrumento adequado para que os alunos possam aprender e desenvolver competências que contribuam para a sua formação (Coutinho et al., 2008).

A programação de robots pode ser uma ferramenta que permite a construção de projetos, de ideias e conceitos, devidamente enquadrados no contexto social dos alunos, e que se relacionam com as suas experiências de vida (Pinto, 2016).

Borges (2012, p. 72) enumera algumas das potencialidades da introdução da robótica no processo educativo:

- "Interdisciplinaridade;

- Motivação por parte dos alunos;

- Estímulo à criatividade e à iniciativa;

- Utilização de metodologias ativas;

- Visualização rápida dos resultados práticos;

- Raciocínio lógico;

- Resolução de problemas;

- Desenvolvimento de pensamento abstrato;

- Construção do conhecimento;

- Partilha e pesquisa de informações a nível global;

- Aplicação prática dos conhecimentos".

A par das imensas potencialidades existem também algumas dificuldades que Ribeiro, Coutinho \& Costa (2011a, p. 440) atribuem "ao tempo de maturação necessário a qualquer tecnologia para ser integrada nas salas de aula". Os mesmos autores destacam ainda mais alguns fatores:

- o carácter técnico da área da Robótica que conduz a algum receio dos professores envolvidos;

- a falta de material de índole pedagógica que tire partido da RE para as diversas áreas curriculares (e.g. manuais, tutoriais);

- a falta de oportunidades para a formação de professores nesta área;

- carência ao nível dos estudos quantitativos que possam concretizar as qualidades pedagógicas desta ferramenta de forma mais evidente" (Ribeiro,

Coutinho \& Costa, 2011a, p. 440).

Outro fator que é muitas vezes referido como dificuldade, prende-se com o investimento que é necessário realizar para adquirir estes equipamentos (Coutinho, Ribeiro \& Costa, 2008).

\section{Principais tipologias de robots}

Existem muitos modelos de robots vendidos prontos a ser utilizados pelo professor, ou pais, em contexto educativo. Em Portugal os mais conhecidos na comunidade educativa são os robots produzidos pela LEGO®. Os equipamentos desta marca permitem que os alunos, do básico ao secundário, personalizem e montem robots, quer a partir dos modelos propostos pela marca, quer, utilizando a criatividade, em propostas concebidas para cada situação-problema apresentada. Outro equipamento muito divulgado é o robot BeeBot ${ }^{\circledR}$ para programação de percursos no $1 .^{\circ}$ ciclo do ensino básico. Este robot é comercializado em duas versões: uma com programação através de botões e outra através de programação com recurso a dispositivos móveis, via bluetooth. Existe também uma utilização crescente de robots desenvolvidos a partir de placas de prototipagem livre (por e.g. o arduino), quer seja em propostas de cariz mais comercial com kits praticamente prontos, quer seja robots idealizados e criados por professores e alunos em salda de aula ou em clubes de robótica. A versatilidade desta solução permite um sem número de propostas adequadas à utilização em contexto educativo, com graus de complexidade muito distintos e com aplicabilidade em diversas áreas do saber.

\section{Estudos analisados}

Para aferir o estado da arte da robótica educativa em Portugal, analisámos textos sobre a temática de programação e robótica presentes nos repositórios das universidades do Minho, Trás-os-Montes e Alto Douro, Porto, Aveiro, Coimbra, Lisboa e Algarve.

Tabela 1.

Tipologias de textos analisados

\begin{tabular}{lc}
\hline Tipologia de publicação & Frequência \\
\hline Publicações em revistas & 1 \\
Publicações em atas de eventos académicos & 7 \\
Capítulos de livros & 1 \\
Dissertações de mestrado & 15 \\
Dissertações de doutoramento & 2 \\
\hline
\end{tabular}

Tabela 2.

Público-alvo das investigações

\begin{tabular}{lc}
\hline Grau de ensino & Frequência \\
\hline Pré-escolar & 3 \\
Primeiro ciclo do ensino básico & 5 \\
Segundo ciclo do ensino básico & 1 \\
Curso de Educação-Formação & 1 \\
Secundário - Cursos Profissionais & 6 \\
Secundário - Aplicações Informáticas B & 3 \\
Alunos com necessidades educativas especiais & 5 \\
\hline
\end{tabular}


Tabela 3.

Disciplinas envolvidas e conteúdos curriculares abordados

\begin{tabular}{lc}
\hline Disciplina & Frequência \\
\hline Linguagens de programação & 2 \\
Português & 2 \\
Matemática & 6 \\
Programação & 2 \\
Aplicações informáticas B & 3 \\
Educação tecnológica & 1 \\
Programação e sistemas de informação & 1 \\
Física & 1 \\
Estudo do meio & 1 \\
Expressões & 1 \\
\hline
\end{tabular}

\section{Questões de investigação}

As questões de investigação são as mais variadas. Em comum, entre as investigações analisadas, estuda-se o papel da robótica educativa nas aprendizagens, na motivação, no desenvolvimento de competências por parte dos alunos. Nos estudos que envolvem alunos com necessidades educativas especiais é, também, abordada a interação dos mesmos com os robots e as suas, eventuais, vantagens. Destacamos, como exemplo, algumas das questões de investigação enunciadas:

- "Será que os alunos consideram que o feedback fornecido pelo robot os ajuda no processo de aprendizagem?" (S. M. C. Ferreira, 2013, p. 59);

- "Será que o ensino-aprendizagem de programação com recurso à Robótica Educativa promove o trabalho colaborativo?" (Celestino, 2013, p.28);

- "Será que a utilização da Robótica Educativa contribui para o cumprimento do objectivo de aprendizagem preconizada na intervenção, ou seja, ensino de estruturas e repetição?" (Celestino, 2013, p.28);

- "Como é que as crianças aprendem a programar em idade pré-escolar?” (Pinto \& Osório, 2015);

- "Pretendeu-se analisar o impacto da robótica educativa na aprendizagem dos alunos" (Oliveira, 2013, p. 66);

- "Can a LEGO robot (simple, lowcost, configurable and modular robot) contribute to promote social interaction and verbal and non-verbal communication in children with ASD?" (S. Costa, Soares, Santos, Pereira \& Moreira, 2016, p. 57);

- "The goal was to work communication and interaction skills, and also to encourage him to ask for the robot's action, promoting joint attention. Case 4 is a boy who has no verbal communication and he performs repetitive motor movements consistently, having low control of his movements. In this way, the goal for Case 4 was to work social interaction and reducing repetitive motor movements over the sessions" (S. Costa et al., 2016, p. 51);

- "Pode o uso de robots, no processo ensino-aprendizagem, proporcionar melhores resultados de aprendizagem relativamente às áreas do Som e da Luz?” (J. M. V. Silva, 2008, p. 52);
- "Pode o uso de robots, no processo ensino-aprendizagem, contribuir para o desenvolvimento de competências do tipo social e atitudinal, nos alunos?" (J. M. V. Silva, 2008, p. 52)".

Condições para a aprendizagem com robots e tipologia de atividades a implementar

Ribeiro et al. (2011b, p. 1501) considera que para que o aprendente possua uma fluência tecnológica e, neste caso, robótica será necessário:

"Aprenda a planificar - o aluno deverá de ser capaz de desenhar o protótipo que pretende ver construído posteriormente para resolver determinadas tarefas e desafios.

Aprenda a programar - através da programação de um robô os alunos estão a construir programas a partir de instruções simples que poderão servir para serem utilizados em acções complexas. O aluno está a aprender a construir e organizar o seu conhecimento.

Aprenda a relacionar - o aluno quando está perante um novo conhecimento deverá ser capaz de relacionar este com o que já possui. Por outro lado, perante a linguagem de programação, que neste caso é icónica, deverá também relacionar os símbolos com as palavras. Reconhecer o significado de cada símbolo para que seja capaz de comunicar com a máquina".

Pinto \& Osório (2016), que investigam a utilização de robots por alunos do pré-escolar, consideram que "nestas atividades, o importante é que a criança possa experimentar, nos primeiros anos de vida, uma ampla variedade de experiências significativas: emocionais, culturais e de todas as áreas de conhecimento. É importante que a criança possa utilizar o seu corpo para se expressar e, inclusive antes de aprender a falar, que também seja capaz de se movimentar no espaço, conhecer os limites e adaptar-se a eles, desenvolvendo competências de orientação especial" (p. 1567).

No que respeita à definição das melhores circunstâncias para utilizar robots em contexto educativo, Santos (2015) considera que "é acompanhando o quotidiano das crianças, que melhor se percebem se definem essas circunstâncias". Ainda o segundo o mesmo autor, neste contexto, conhecem-se "as suas possibilidades e de que forma pode ser uma mais-valia para a motivação, interesse e concentração das crianças. Não é contudo possível dar receitas pois o que se aplica em determinado tempo e espaço com um grupo específico pode não ser o mais adequado noutro enquadramento" (p. 351).

\section{Resultados}

A maioria dos estudos analisados identifica vantagens na utilização da robótica educativa com os alunos, algumas das quais explanamos seguidamente.

Mas outros estudos existem que, apesar de não conclusivos, levam os autores a considerar que: "apesar de não poder afirmar que a utilização da Robótica Educativa, utilizando-se robôs da Lego ${ }^{\circledR}$ Mindstorms ${ }^{\circledR}$ NXT 2.0, contribui para a consolidação de conceitos de programação, considero que poderá ser uma alternativa para o ensino de alguns conceitos de programação e a 
alunos que estejam a iniciar-se na programação. Para além disso, a sua utilização em conjunto com a Aprendizagem Baseada em Problemas, permitem contextualizar as aprendizagens dos alunos, principalmente em alunos de cursos profissionais" (Ressurreição, 2012, p. 92); e, "analisando todos os dados recolhidos não é possível retirar conclusões pertinentes sobre o impacto da robótica educativa na programação devido ao número de participante e tempo de intervenção. Contudo há evidências de resultados positivos na sua utilização no âmbito da concretização de soluções e criação de algoritmos mais precisos. Os alunos que participaram no estudo evidenciaram melhorias na criação de algoritmos que poderão advir da experiência da programação com robôs melhorias nas aprendizagens" (J. M. de A. Costa, 2012, p. 38).

Primeiro ciclo. "No que diz respeito ao desenvolvimento da orientação espacial, nomeadamente das capacidades de lateralização e de lateralidade, as atividades desenvolvidas com robôs levaram as crianças a reconhecer, organizar e sintetizar as informações oriundas do meio à sua volta, proporcionando um momento onde estas estiveram a movimentar-se e a localizar objetos, neste caso as serras de Portugal tendo como base pontos de referência. Esta experiência realizada com os alunos demonstrou que o trabalho de grupo permitiu momentos de comunicação, tanto de raciocínios como de ideias matemáticas, oralmente e por escrito, de autoconfiança, de criatividade, de rotinas de trabalho e de persistência, facilitando o desenvolvimento destas competências" (Carmo, 2013, p. 65).

Ensino da Física. “(...) a Robótica constitui-se com uma mais-valia para o ensino da Física que, por tudo que foi exposto nesta dissertação, não deve ser desperdiçada" (J. M. V. Silva, 2008, p. 53).

Ensino da Programação. "A robótica educativa pode, de facto, ser utilizada na consolidação de conhecimentos numa fase mais avançada das aprendizagens dos alunos em programação e não apenas numa fase inicial, mas não se pode deixar que os alunos explorem os recursos sem apoio e orientação vincadamente marcada pelos objetivos do professor" (Bernardo, 2012, p. 99).

Ensino com alunos com Perturbações do Espectro do Autismo (PEA). "O impacto do robô nas crianças não é facilmente comprovado, sendo percetível que o interesse em relação ao robô depende da criança em questão e esse fator condiciona o sucesso do estudo. Foi possível observar que nem todas as crianças foram capazes de adquirir a competência definida com a introdução do robô, porém a maioria delas desenvolveu alguma capacidade, seja ela a competência pré-definida, o contato visual ou o tempo de permanência na atividade. Assim, pode afirmar-se que o robô pode ser um facilitador/mediador interessante na interação com crianças com PEA, bem como pode ajudar a captar a atenção e manter a concentração das mesmas na atividade, promovendo interações triádicas e conduzindo a que estas crianças adquiram novas competências, de forma a melhorar a sua qualidade de vida" (S. I. A. da Silva, 2012, pp. 101-102).
Motivação. "Mas é inegável que se trata de uma actividade que motiva os alunos e foi já demonstrado que tem fundamentos pedagógicos sérios e que contribui para o adquirir de competências em áreas curriculares chave dos curricula do Ensino Básico" (C. Ribeiro, 2006, p. 122).

\section{Conclusões}

A preparação das atividades é muito importante, tal como refere Bernardo (2012, p.99) “(...) para tirar o máximo proveito pedagógico da utilização de robôs é necessário despender algum tempo antecipadamente e organizar muito bem a forma de orientação a dar aos alunos".

Temos ainda que referir, tal como se passa com as outras tecnologias de informação e comunicação, a robótica não constitui panaceia para os problemas da educação a nenhum nível (C. Ribeiro, 2006). É, no entanto, uma ferramenta com enorme potencial que permite, quando adequadamente utilizada, promover a motivação e a qualidade das aprendizagens dos alunos. Dada a sua versatilidade, pode o mesmo equipamento, ser utilizado em múltiplos contextos educativos, em diversas áreas disciplinares ou em contexto inter e transdisciplinar.

Importa pois que se identifiquem as melhores práticas, os equipamentos mais adequados a cada contexto e a cada atividade e os conteúdos mais relevantes para obter os melhores resultados.

\section{Referências}

Almeida, C. M. dos S. de. (2015). A importância da aprendizagem da robótica no desenvolvimento do pensamento computacional: um estudo com alunos do 4. ${ }^{\circ}$ ano (Mestrado). Universidade de Lisboa, Lisboa.

Almeida, L., Azevedo, J., Ribeiro, F., Cardeira, C., Lima, P., Fonseca, P., \& Santos, V. (2001). Robótica 2001 Festival Nacional de Robótica. Robótica, (64), 64-65.

Bernardo, M. P. (2012). A robótica educativa aplicada na consolidação de conhecimentos na disciplina de Linguagens de Programação (Mestrado). Universidade de Lisboa, Lisboa.

Borges, A. P. R. P. (2012). Uma experiência educativa com robótica inteligente (Mestrado). Universidade do Minho, Braga.

Carmo, B. S. do. (2013). A robótica educativa no desenvolvimento do raciocínio matemático (Mestrado). Universidade do Algarve, Faro.

Celestino, H. A. D. S. (2013). Aprendizagem de estruturas de controlo com recurso à robótica educativa (Mestrado). Universidade de Lisboa, Lisboa.

Conchinha, C., Freitas, J. C. de, Cleto, B. G., Silva, S. G., \& Moraes, A. de. (2016). Robótica virtual o robótica tangible: Un estudio sobre el potencial inclusivo de la programación y robótica. In Virtual USATIC'16 Ubicuo y Social: Aprendizaje con TIC. Zaragoça: Universidad de Zaragoza.

Conchinha, C., Freitas, J. C. de, \& D'Abreu2, J. V. V. (2014). A comunidade Robots \& NEE A robótica educativa, um ambiente inclusivo. In A comunidade Robots \& NEE A robótica educativa, um ambiente 
inclusivo (pp. 1464-1468). Lisboa, Portugal: Instituto de Educação da Universidade de Lisboa.

Costa, J. M. de A. (2012). A robótica educativa na aprendizagem da programação (Mestrado). Universidade de Lisboa, Lisboa.

Costa, S., Soares, F., Santos, C., Pereira, A. P., \& Moreira, M. F. (2016). Robô Lego \& Perturbações do Espectro do Autismo: Uma potencial parceria? Revista de estudios e investigación en psicologia y educación, 3(1), 50-58.

Coutinho, C. P., Ribeiro, C., \& Costa, M. F. (2008). RobôCarochinha : um estudo sobre robótica educativa no ensino básico. In DIAS, P. ; OSÓRIO, A. J., org. Ambientes educativos emergentes (p. 35-55.). Braga: Centro de Competência da Universidade do Minho.

Ferreira, H. J. D. (2013). Os robôs no enino da programação (Mestrado). Universidade de Lisboa, Lisboa.

Ferreira, S. M. C. (2013). A robótica educativa no ensino-aprendizagem de estruturas de seleção (Mestrado). Universidade de Lisboa, Lisboa, Portugal.

Gonçalves, F. D. da C. G. (2012). Utilização de robótica educativa no ensino de programação a alunos do secundário (Mestrado). Universidade de Lisboa, Lisboa.

Norte, S. (2010). Interfaces pessoa-máquina em ambientes de programação LOGO (Doutoramento). Universidade do Algarve, Faro.

Oliveira, D. F. R. (2013). A Robótica Educativa no Ensino e Aprendizagem de Conceitos de Programação e Algoritmos (Mestrado). Universidade de Lisboa.

Pinto, M. S. M. (2016). Desafíos de Programación y Robótica en Educación Preescolar: Proyecto Kids Media Lab. In Tecnología, innovación e investigación en los procesos de enseñanza-aprendizaje (pp. 18481855). Barcelona.

Pinto, M. S. M., \& Osório, A. M. (2015). Tecnologias e Aprendizagem de Programação em Idade Pré-escolar: Projeto Kids Media Lab. In I Encontro Professores Inovadores com TIC. Bragança.

Pinto, M. S. M., \& Osório, A. M. (2016). As TIC em contexto de educação de infância: atividades sobre pensamento computacional e programação. In XIII CONGRESSO SPCE, 2016 (pp. 1565-1571). SPCE.

Ressurreição, R. P. L. (2012). A consolidação de conceitos de programação utilizando a robótica educativa (Mestrado). Universidade de Lisboa, Lisboa.

Ribeiro, C. (2006). RobôCarochinha: Um Estudo Qualitativo sobre a Robótica Educativa no 1o ciclo do Ensino Básico (Mestrado). Universidade do Minho, Braga.

Ribeiro, C., Coutinho, C. P., \& Costa, M. F. (2011a). A Robótica Educativa como Ferramenta Pedagógica na Resolução de Problemas de Matemática no Ensino Básico. In Conferência Ibérica de Sistemas e Tecnologias da Informação (pp. 440-445). Vila Real.

Ribeiro, C., Coutinho, C. P., \& Costa, M. F. (2011b). ROBOWIKI: Um recurso para a robótica educativa em língua portuguesa. In VII Conferência Internacional de TIC na Educação (pp. 1499-1514). Braga: Universidade do Minho. Instituto de Educação. http://hdl.handle.net/1822/12821
SANTOS, C. S. D. (2015). Enquadramento de atividades de programação com robôs de solo na educação pré-escolar (Doutoramento). Universidade de Trás os Montes e Alto Douro, Vila Real.

Silva, S. I. A. da. (2012). Estudo do efeito da utilização de uma plataforma robótica na intervenção em crianças com perturbações do espetro do autismo (Mestrado). Universidade do Minho, Braga.

Silva, J. M. V. (2008). Robótica no ensino da Física (Mestrado). Universidade do Minho, Braga.

Varanda, F. M. S. P. D. (2012). Robótica educativa no ensino de subprogramas (Mestrado). Universidade de Lisboa, Lisboa. 